\title{
Autour DE LA NOTION DE SACER
}

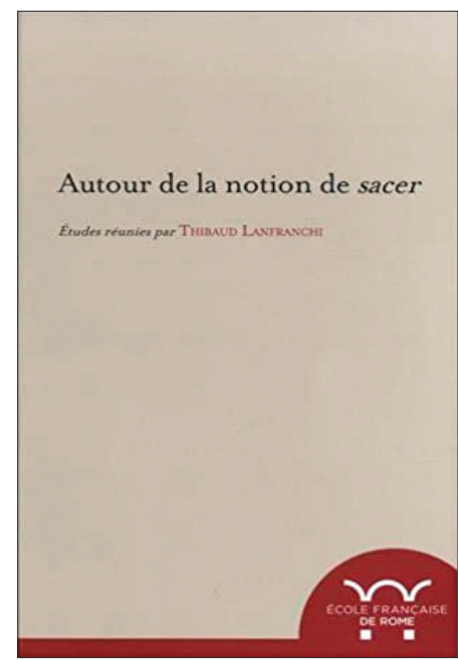

LANFRANCHI, THIBAUD (ed.). (2018). Autour de la notion de sacer. Rome: École française de Rome. 300 pp., 43,06€ [ISBN 978-2-72831288-7].

\section{FABIO GIORGIO CAVALLERO \\ Sapienza Università di Roma - Scuola Archeologica Italiana di Atene FABIOGIORGIOCAVALLERO@GMAIL.COM}

AUtOUR DE LA NOTION DE SACER È UNA PREgeVole RACCOLTA DI saggi frutto di una giornata di studi svoltasi nell'aprile 2014 all'École française de Rome. L'iniziativa, promossa da Thibaud Lanfranchi, era inserita all' interno del progetto di ricerca Italia Picta (2012-2016), per lo studio dei rapporti tra Roma e i popoli dell' Italia antica durante il periodo della conquista (VI-III sec. a.C.). Tali relazioni sono state analizzate non soltanto dal punto di vista romano, ma anche dal punto di vista dei popoli "vicini" al fine di comprendere come le conquiste territoriali dell' Urbe siano state utilizzate per l'acquisizione di esperienze successivamente reinvestite nell'amministrazione dell' Italia. Si tratta di un tema fondamentale per la comprensione dell'espansionismo romano e delle sue origini poiché è proprio nei secoli in questione che Roma elaborò e concepì alcuni dei più importanti strumenti politico-amministrativi che successivamente le permisero di organizzare il suo dominio sul resto della penisola italiana, 
delle Province e dell' Impero Mediterraneo. Il volume ha dunque innanzitutto il pregio di affrontare aspetti fondamentali per la storia del Mediterraneo antico, partendo inoltre da una premessa metodologica fondamentale: non è più possibile, come accaduto in passato, affrontare tematiche relative al mondo classico senza impiegare un approccio multidisciplinare. Per questo motivo la giornata di studi, all'origine del volume, ha previsto otto interventi di linguisti, archeologi, antichisti, romanisti e storici. Ricerche come quella intorno al significato di sacer devono dunque essere affrontate da diversi orizzonti e i processi emergenti dalle diverse serie documentali possono essere confrontati al fine di ricostruire una storia che, nel caso specifico, è quella del significato di un termine il quale, come giustamente sottolineato da A. Bertrand (p. 241), gli antichi hanno sempre cercato di ridefinire nel tempo, anche in relazione ad altra terminologia giuridico-sacrale appartenente alla stessa sfera semantica. E l'esperienza giuridica romana è certamente, sotto questo punto di vista, quella che attesta in maniera più chiara questa mutabilità permettendo anche di riflettere sulle insidie di una visione statica del mondo antico e delle sue Istituzioni. Come è noto, infatti, il materiale utile a un tentativo di definizione della nozione di sacro nella cultura latina è quello più abbondante ed è riconducibile in particolar modo all'esperienza giuridica romana. È per questo motivo che, muovendosi in tale ambito, si possono tentare operazioni di definizione più raffinate rispetto a quelle che è possibile elaborare per altri contesti culturali la cui tradizione letteraria e giuridica è andata irrimediabilmente perduta. Non stupisce pertanto che la romanistica si sia confrontata, in particolar modo tra diciannovesimo e ventesimo secolo, non soltanto con il problema relativo al significato giuridico di sacer, ma anche con quello del rapporto che questa particolare "qualità" - attribuibile a loca, res, etc. (v. infra) - intratteneva con altri termini utilizzati in ambito giuridico-sacrale, fra tutti sanctus (di questo si occupa il contributo di Elena Tassi Scandone, "Sacer e sanctus: quali rapporti?", pp. 131-170). I diversi studi, tuttavia, date le difficoltà nell'armonizzare le differenti definizioni date dai giuristi antichi, hanno spesso ammesso una generale incoerenza sia tra le fonti a nostra disposizione sia all'interno dell'opera di uno stesso autore. Esemplificative in questo senso sono le conclusioni di Ferdinando Bona ([1987]. Alla ricerca del De verborum, quae ad ius civile pertinent, significatione di Elio Gallo. 1. La struttura dell'opera. BIDR, 90, pp. 119-168) in merito al noto passo di E. Gallo riportato in Fest. 348 L., considerato dall' illustre studioso troppo contradditorio per non intravedere un intervento di V. Flacco che ne avrebbe minato la genuinità originaria lasciando nel testo cesure e aporie dovute alle diverse impostazioni delle opere dei due autori (divisa per genera quella di E. Gallo, ordinata alfabeticamente quella di V. Flacco). Si è così giunti, in alcuni casi, a considerare come del tutto inattendibili le testimonianze degli antichi in materia di res sanctae rinunciando per questo a 
ricostruire i rapporti tra alcuni istituti appartenenti al ius divinum (v. in particolare Fantetti, E. [1956]. L'inquadramento classico delle res sanctae. Labeo, 2, p. 94). Simili conclusioni, tuttavia, potrebbero dipendere da un "approccio di tipo dogmatico e perciò stesso essenzialmente statico" delle ricerche (p. 169) ed è per questo che risulta necessario applicare una prospettiva dinamica (ossia diacronica e non sincronica) allo studio dei singoli istituti giuridici, e in particolar modo per quelli che "affondano le radici nel diritto della Roma monarchica o, addirittura, nei mores delle comunità preciviche" (p. 168). Diventa in questo modo possibile tentare definirne le trasformazioni da essi subite nel corso dei secoli e comprenderne, laddove possibile, le cause.

Un'operazione, questa, aggiungo, resa possibile dal riconoscimento di una stratificazione che non può più essere - come spesso accaduto - ignorata, dal momento che riposa sulla tendenza antica di mantenere attivi, seppur sclerotizzandoli, istituti ormai superati "grazie alla straordinaria capacità della giurisprudenza romana di elaborare soluzioni innovative per rispondere alle esigenze...di una società in continua evoluzione" (caso emblematico, in questo senso, è quello del mantenimento di alcune funzioni dei comitia curiata nonostante la creazione della più recente assemblea centuriata). D'altra parte, non è cosa sconosciuta che la gestualità e la ritualità permisero la conservazione di formule e pratiche per così lungo tempo che, a partire da un certo momento, esse non furono più neppure comprese (pur preservandone comunque la trasmissione).

Di questo rinnovato metodo è un chiaro esempio anche lo studio di Roberto Fiori sulla condizione di homo sacer nel mondo romano (pp. 171-228), tema da sempre al centro del dibattito scientifico e ricco delle più diverse interpretazioni. Le quali, tuttavia, sembrano perdere la loro ragion d'essere proprio nel momento in cui si ammette che "il presupposto fondamentale (delle precedenti interpretazioni) è....una concezione unitaria del sacrum che identifica il regime degli homines sacri e delle res sacrae, sul presupposto che 'sacer è ciò che appartiene al dio'... Tuttavia ci si accorge del fatto che una simile concezione unitaria non può essere ammessa". Ora, al di là delle varie conclusioni alle quali si potrebbe giungere, importa sottolineare in particolar modo un preciso aspetto del metodo impiegato dall'A. Anche in questo caso, infatti, un'attenta rivalutazione di tutte le fonti a disposizione in chiave diacronica e non in una visione "codicistica", figlia della dottrina ottocentesca, ha permesso a R. Fiori - che certamente ha il merito di aver lavorato di bulino di bulino su diversi brani (e.g. il notissimo Fest. 442 e 424 L.) - di giungere non soltanto alla ricostruzione di un importante Istituto del mondo romano arcaico, ma anche alla comprensione della posizione assunta da un individuo colpito dalla sacratio nei confronti della comunità di appartenenza. E per converso, proprio il lavoro di scavo nelle fonti ha permesso di scardinare la visione di una sacratio il cui valore sarebbe variato nel V secolo a.C. 
in seguito ai giuramenti collettivi prestati dai romani "in occasione della cacciata dei Tarquini e della secessione plebea del 494 a.C.. Una prospettiva diacronica di studio dunque che, contraddicendo in sostanza ipotesi che prevedevano una variabilità nel tempo di un Istituto, conferma la bontà del metodo impiegato.

Le fonti, dunque, di qualsiasi tipo esse siano, possono e devono essere indagate anche stratigraficamente, così come, d'altronde, già nel 1959 auspicava Pietro de Francisci: "la ricostruzione delle istituzioni primitive esige un lavoro di scavo: e, quando sia ben condotto...si può sempre sperare di poter recare qualche nuovo contributo alla conoscenza della preistoria o della protostoria delle istituzioni" (Primordia Civitatis). Proprio un attento lavoro di scavo è stato compiuto con questo volume che permette di superare alcune delle apparenti contraddizioni spesso indicate nella storia degli studi come indizi di falsificazioni, incomprensioni e incoerenze. Tale sguardo diacronico e per differentiam è certamente, al di là delle differenti tematiche affrontate, un importante contributo di questa raccolta di studi che ha anche il merito di proporre alcune motivazioni che sarebbero alla base delle singole "trasformazioni" semantiche. Un esempio su tutti è quello, forse il più significativo, che vede, probabilmente in età giustinianea e in conseguenza di un cambiamento avvenuto nell'epoca classica, le res sanctae passare dalla categoria giuridica normata dal ius divinum a quella delle res publici iuris: un ottimo esempio di come la metodologia di scavo stratigrafico nelle fonti antiche possa condurre a un "nuovo contributo alla conoscenza" della storia tanto dei valori semantici impiegati dagli antichi, quanto del più ampio sistema nel quale essi erano inseriti.

Il volume, grazie ai diversi contributi, assolve esattamente a questi compito dando nuovo stimolo alle ricerche inerenti alla categoria delle cose sacre e delle loro definizioni che spesso si rincorrono su una medesima radice cambiando però di significato e mettendo così in guardia, come ben sottolineato da A. Bertrand, da comparazioni semplicistiche che spesso conducono a traduzioni affrettate e, ancor peggio, errate. Per questo, i contributi di Valentina Belfiore sulla nozione di sacer in etrusco (pp. 40-60), di Emmanuel Dupraz sulla corrispondenza dello stesso termine sulle tavole eugubine (pp. 62-92) e di Giovanna Rocca su sacer nelle iscrizioni umbre (pp. 93-114), sono la dimostrazione non soltanto dell' importanza della ricostruzione di processi ricavabili da serie documentali provenienti da diversi ambiti geografici ma, come giustamente ammesso da Danièle Dehouve nel suo interessante studio "Sacer et Sacré. Notion emic et catégorie anthropologique" (pp. 16-37), anche e soprattutto, della loro "comparazione" al fine di chiarire sfumature semantiche che altrimenti resterebbero nell'ombra oppure verrebbero archiviate nella categoria dell' "impossibile da comprendere" o, peggio ancora, del tutto fraintese. Emblematiche in questo senso sono le parole di A. Bertrand su parte dello studio di Dupraz sui termini impiegati in 
umbro: "Si on a pu traduire l'adjectif substantivé sakref par "porcelets», sur la base des rares attestations de sacris en latin, plusieurs contextes d'emploi invitent à prolonger la réflexion pour faire ressortir la richesse des traits sémantiques de l'adjectif".

Uno dei pericoli nell'affrontare temi che dipendono, per ovvie ragioni, dall'analisi delle fonti letterarie ed epigrafiche è sottovalutare un aspetto fondamentale ossia quello legato agli spazi che queste descrivono. Il rischio è infatti quello di elaborare lodevoli e articolate costruzioni teoriche di Istituti o categorie antiche che mancano però di riscontro nei resti provenienti dallo stesso mondo che le ha elaborate e trasmesse. Nel volume in questione, tuttavia, il contributo di Olivier de Cazanove mostra come anche questo importante aspetto sia stato considerato. L'A. infatti, partendo - giustamente - dal valore semantico del termine sakaraklùm (attestato sul cippo abelano), tenta di definire quale e di che tipo fosse lo spazio definito da uno dei cippi che evidentemente dovevano circondare un tempio o un santuario ("Le sacré en Partage. Sakaralùm, temple ou sanctuarie sur le cippe d'Abella?”, pp. 113-130). Anche in questo caso, quindi, la comparazione di serie documentarie diverse dimostra ancora una volta tutto il suo potenziale euristico. Partendo da Partendo da quest' ultima osservazione si potrebbe ammettere che poteva forse essere approfondita la questione dello spazio sacro romano. Ma ciò dipende certamente dal fatto che le moderne strategie editoriali non lasciano più grandi spazi di manovra e i volumi devono pur essere chiusi in un numero contingentato di pagine. Nel caso de Autour de la notion de sacer, tuttavia, ciò è bastato non soltanto ad affrontare diverse questioni che negli anni si erano gordianamente annodate ma anche a suggerire spunti di ricerca che potranno chiarire le numerose zone d'ombra che ancora avvolgono diverse tematiche inerenti alla nozione di sacer.

Per concludere: si tratta di un volume assai complesso e articolato che, per ricchezza dei contenuti e dei punti di vista, offre numerose e interessanti occasioni di riflessione e discussione. Per darne conto in maniera adeguata occorrerebbe ben altro spazio di quello concesso a questa breve considerazione. Io ho voluto sottolineare in particolare un aspetto tra $\mathrm{i}$ tanti possibili: il valore aggiunto rappresentato da un metodo che predilige l'analisi diacronica di tutte le fonti per ricostruire la storia di Istituti e valori semantici, in questo caso specifico legati a una particolare condizione giuridico-sacrale. In primo luogo ciò è naturale per un archeologo come me, abituato a conoscere il mondo antico ricostruendo per prima cosa il divenire di oggetti, stratificazioni e strutture, paesaggi. Ma c'è di più. Tale approccio consente infatti di raggiungere due obiettivi fondamentali per una visione realmente interdisciplinare. $\mathrm{Da}$ un punto di vista più generale, le diverse discipline/filologie - in questo caso storia del diritto, filologia e archeologia - creano serie di informazioni e teorie impiegando un metodo analogo, che possiamo definire stratigrafico. Esse pertanto consentono 
di comparare efficacemente processi ricostruiti in modo autonomo e in base a serie di documenti diversi. Tutto ciò non può che arricchire il dibattito scientifico, troppo spesso condizionato da eccessivi specialismi. Infatti, operando in questo modo, emerge la capacità di tradurre materie specifiche in sistemi di conoscenze riconducibili alla ricerca storica e condivisibili, anche se scaturiti da diversi "punti di accesso" al complesso dei dati/documenti. Inoltre, da un punto di vista più specifico, la possibilità di ricostruire Istituti e valori semantici nel loro divenire storico rivela, non senza buone dosi di stupore e voluttà generate dalla vividezza dei risultati ottenuti, quanto indietro nel tempo possano risalire e con quale grado di persistenza siano state conservate le le antiche istituzioni giuridico-sacrali. Il suolo di Roma ha rivelato una storia molto più articolata e antica di quanto si credesse fino a non molti anni fa, e lo ha fatto perché quella terra è stata scavata stratigraficamente. Allo stesso modo, le fonti scritte e le memorie che esse conservano rivelano una "profondità storica" e una "storia" raggiungibile soltanto a patto di sottoporle a un analogo "lavoro di scavo". 